\title{
Leptin : adiponectin ratio as an atherosclerotic index in patients with type 2 diabetes: relationship of the index to carotid intima-media thickness
}

Received: 13 July 2005 / Accepted: 22 August 2005 / Published online: 1 November 2005

(C) Springer-Verlag 2005

\begin{abstract}
Abbreviations BP: Blood pressure - CIMT: Carotid intima-media thickness $\cdot \mathrm{L}: \mathrm{A}$ : Leptin: adiponectin ratio
\end{abstract}

To the Editor: Diabetic metabolic abnormalities induce vascular dysfunction that predisposes diabetic patients to atherosclerosis [1]. Complications of atherosclerosis cause much of the disability and most of the mortality in diabetic patients [1]. Clinical atherosclerotic manifestations in diabetes occur primarily in vascular beds such as extracranial carotid arteries and coronary arteries [1]. On the other hand, two adipocytokines, leptin and adiponectin, play important roles in the metabolic regulation of obesity and obesity-related complications [2]. In obese subjects, plasma leptin concentrations are elevated and adiponectin concentrations decreased. Consequently, it is speculated that leptin could accelerate and adiponectin restrain the development of atherosclerosis [2]. Recently, Satoh et al. [2] reported that the plasma leptin : adiponectin ratio (L : A) is correlated with pulse wave velocity values and may be a useful biomarker for atherogenesis in obese type 2 diabetic patients. However, the significance of the $\mathrm{L}: \mathrm{A}$ in diabetes has still not been thoroughly investigated.

Considerable research has established carotid intimamedia thickness (CIMT) as a clinical surrogate marker of atherosclerosis [3]. Although pulse wave velocity values can be roughly correlated with CIMT [3], when assessing

K. Kotani $(\bowtie) \cdot$ K. Saiga $\cdot$ Y. Kurozawa

Division of Health Administration and Promotion,

Tottori University,

86 Nishi-cho,

Yonago, 683-8503, Japan

e-mail: kakotani@grape.med.tottori-u.ac.jp

Tel.: +81-859-348026

Fax: $+81-859-348085$

N. Sakane

Department of Preventive Medicine,

Clinical Research Center for Endocrine and Metabolic Disease,

National Hospital Organization Kyoto Medical Center,

Kyoto, Japan pulse wave velocity in clinical practice, confounding factors such as abnormalities of blood pressure (BP), blood flow and sympathetic tones might increase the variability of pulse wave velocity [4] that is often observed in subjects with obesity and type 2 diabetes. Also, atherosclerosis involves two different processes: atherosis and sclerosis of the arterial wall [3]. CIMT seems to reflect atherotic (structural) changes more accurately than pulse wave velocity values, whereas the latter is considered as more of a reflection of sclerotic (functional) changes [3, 5]. Type 2 diabetes accelerates both types of change, whereas hyperlipidaemia causes mostly atherotic changes [3]. In another study using CIMT measurements [6], type 2 diabetes caused atherosclerosis at a more accelerated rate than hypercholesterolaemia, suggesting an important role of CIMT as a surrogate of atherosclerosis in diabetes. It is therefore crucial to determine whether the findings of Satoh et al. [2] are replicable with regard to CIMT measurement. With these results in mind, we examined the relationship between L : A and CIMT in patients with diabetes.

A total of 152 Japanese type 2 diabetic patients (73 men, 79 women; mean age $=70.1 \pm 8.7$ years [mean \pm standard deviation]), with stable conditions, and treated with diet and/or sulfonylureas, were enrolled for this study. Sixtythree subjects were treated with sulfonylureas and 89 were treated with diet only. The study was approved by the institutional ethics committee and each subject gave informed consent. Smokers were defined as current smokers. Of the smokers, 39 were men and 10 were women. Overnight fasting plasma insulin, lipid panels and blood $\mathrm{HbA}_{1} \mathrm{c}$ values were measured by the standard procedures. Plasma leptin levels were measured using a commercially available enzyme immunoassay kit (Cayman Chemical Company, Ann Arbor, MI, USA), and adiponectin levels were measured with an enzyme-linked immunosorbent assay kit (Otsuka Pharmaceutical, Tokyo, Japan). We measured BP in the right upper arm of seated patients using a standard sphygmomanometer. CIMT was determined using a Bmode ultrasound scanner (SSD-900; Aloka, Tokyo, Japan) 
and a 7.5-MHz linear probe with subjects in the supine position. Because the internal and external carotid arteries could not be fully detected in many patients, the intimamedia thickness of the common carotid artery was bilaterally measured with longitudinal and transverse scans in segments that were free of plaque, plaque being defined as the presence of wall thickening at least $50 \%$ greater than the adjacent thickness. Three measurements of CIMT were taken: one at the thickest site and one at two other points, $1 \mathrm{~cm}$ upstream and $1 \mathrm{~cm}$ downstream from the thickest site. These measurements were averaged. All assessments were made by a trained specialist with more than 10 years' experience and no knowledge of the subjects' clinical characteristics. In all statistical analyses, differences were considered significant at $p<0.05$.

The average levels of each parameter in all patients were as follows: BMI, $23.8 \pm 2.50 \mathrm{~kg} / \mathrm{m}^{2}$; systolic BP, $143.6 \pm$ $14.3 \mathrm{mmHg}$; diastolic BP, $80.7 \pm 7.9 \mathrm{mmHg}$; total cholesterol, $5.13 \pm 0.76 \mathrm{mmol} / \mathrm{l}$; triglycerides, $1.32 \pm 0.66 \mathrm{mmol} / \mathrm{l}$; high-density lipoprotein cholesterol, $1.44 \pm 0.41 \mathrm{mmol} / \mathrm{l}$; glucose, $7.00 \pm 1.60 \mathrm{mmol} / \mathrm{l}$; insulin, $8.68 \pm 8.67 \mu \mathrm{U} / \mathrm{ml}$; $\mathrm{HbA}_{1} \mathrm{c}, 7.2 \pm 1.7 \%$; leptin, $23.89 \pm 2.51 \mathrm{ng} / \mathrm{ml}$; adiponectin, $5.60 \pm 2.73 \mu \mathrm{g} / \mathrm{ml}$; L : A, $1.62 \pm 2.41$; CIMT, $0.95 \pm 0.25 \mathrm{~mm}$. The relationships of CIMT levels with age, sex, presence or absence of smoking, BMI, BP, lipid panels, glucose, insulin, $\mathrm{HbA}_{1} \mathrm{c}$ and $\mathrm{L}$ : A were examined using Spearman's rank correlation. These analyses only demonstrated a significant relationship between CIMT and age $(r=0.381$, $p<0.001)$. The relationships between CIMT and the other parameters were not significant. Additionally, when the relationships between CIMT and leptin or adiponectin alone were analysed, adiponectin was weakly but significantly correlated with CIMT $(r=-0.187, p<0.05)$, but leptin was not correlated. When multiple regression analysis for CIMT levels as a dependent variable was performed with the factors described above as explanatory variables, L : A was a significant and independent factor, along with age (Table 1). In a subanalysis of the multiple regression analysis that divided all of the subjects into two groups (group 1: 70 years of age or younger; group 2: 71 years of age or older) according to their age in the equation, $\mathrm{L}: \mathrm{A}$ was a significant and independent factor $(\beta=0.421$, $p<0.001)$, along with smoking status $(\beta=0.270, p<0.05)$ in the younger group $(n=71)$. In the older group $(n=81), L$ : A was not a significant factor, while age was a significant risk for CIMT levels $(\beta=0.268, p<0.05)$.

In this study, an association between CIMT and age was observed, consistent with earlier findings that CIMT increases with ageing [7, 8]. As shown in the younger group, smoking status was also a risk for increased CIMT levels, as previously reported $[3,8]$. The lack of correlation of BP and lipids with CIMT levels may be accounted for by the fact that, even if CIMT predicts cardiovascular disease [3], traditional cardiovascular risk factors such as BP and lipids are insufficiently associated during the progression of CIMT or development of cardiovascular disease, depending on the circumstances $[9,10]$. Leptin and adiponectin might be more relevant to increased CIMT than BP and lipid levels, as in our multiple regression analysis of the whole population, the L : A, similar to age, was a strong indicator of increased CIMT.

Generally, in overweight and obese people, leptin levels are higher and adiponectin levels are lower and thus the $\mathrm{L}$ : A may be relatively high [2]. Furthermore, because leptin and adiponectin could be individually associated with the development of atherosclerosis $[2,11]$, the combination of leptin and adiponectin might effect more atherosclerotic manifestations than would leptin or adiponectin alone [2]. Although our results appeared to support this idea, the relationship of the L : A to CIMT was evident regardless of BMI, contrary to the results of Satoh et al. [2]. This discrepancy could have been influenced not only by the different examination methods [3], but also by the different populations studied. Satoh et al. [2] examined a population with an average age of 59.8 years, more than 10 years lower than in our study. In fact, our subanalysis showed that the L : A had an impact on CIMT in the younger subjects (70 years or younger). Among the older diabetic subjects, there was a tendency for BMI to decline [12]. The relationship between the L : A and BMI may thus be significant in the relatively younger population. Moreover, leptin and adiponectin might have meaningful effects not only at higher levels, as in an obese population, but also at lower levels near the normal range, as in our study population. Although fat distribution and nutritional status were not measured in our study, such factors could also influence the inconsistencies noted. Studies including these parameters are forthcoming.

In conclusion, our study suggests that the $\mathrm{L}$ : A can serve as a clinical marker of atherosclerosis in type 2 diabetic subjects, especially those who are 70 years old or younger. This effect is independent of obesity, partially confirming the proposal by Satoh et al. [2], even though their examination methods and their detectable pathophysiological significance were different. The potential clinical

Table 1 Multiple regression analysis for carotid intima-media thickness with clinical parameters and leptin-to-adiponectin ratio

\begin{tabular}{lcc}
\hline Parameter & $\beta$ & $p$ value \\
\hline Age (years) & 0.365 & $<0.001 *$ \\
Sex, male & 0.100 & 0.242 \\
Smoking, current smoking & 0.112 & 0.208 \\
Body mass index $\left(\mathrm{kg} / \mathrm{m}^{2}\right)$ & 0.043 & 0.571 \\
Systolic BP $(\mathrm{mmHg})$ & 0.022 & 0.785 \\
Diastolic BP $(\mathrm{mmHg})$ & -0.065 & 0.427 \\
Total cholesterol $(\mathrm{mmol} / \mathrm{l})$ & 0.111 & 0.166 \\
Triglycerides $(\mathrm{mmol} / \mathrm{l})$ & 0.127 & 0.105 \\
HDL cholesterol $(\mathrm{mmol} / \mathrm{l})$ & 0.089 & 0.267 \\
Glucose $(\mathrm{mmol} / \mathrm{l})$ & 0.099 & 0.198 \\
Insulin $(\mu \mathrm{U} / \mathrm{ml})$ & 0.023 & 0.769 \\
HbA ${ }_{1} \mathrm{c}(\%)$ & -0.023 & 0.719 \\
Leptin : adiponectin ratio & 0.256 & $0.001 *$ \\
\hline
\end{tabular}

BP, blood pressure; $\beta$, standard regression coefficient. * Meets criterion for statistical significance, set at $p<0.05$ 
contribution of the $\mathrm{L}: \mathrm{A}$, as an easy measurement, to management strategies for atherosclerotic disease among type 2 diabetic patients warrants further study.

Acknowledgements This study was supported in part by a grantin-aid from the Foundation for the Development of the Community, Japan.

\section{References}

1. Beckman JA, Creager MA, Libby P (2002) Diabetes and atherosclerosis: epidemiology, pathophysiology, and management. JAMA 287:2570-2581

2. Satoh N, Naruse M, Usui T et al (2004) Leptin-to-adiponectin ratio as a potential atherogenic index in obese type 2 diabetic patients. Diabetes Care 27:2488-2490

3. Taniwaki H, Kawagishi T, Emoto M et al (1999) Correlation between the intima-media thickness of the carotid artery and aortic pulse-wave velocity in patients with type 2 diabetes. Diabetes Care 22:1851-1857

4. Yamashina A, Tomiyama H, Takeda K et al (2002) Validity, reproducibility, and clinical significance of noninvasive brachial-ankle pulse wave velocity measurement. Hypertens Res 25:359-364

5. Oren A, Vos LE, Uiterwaal CS, Grobbee DE, Bots ML (2003) Aortic stiffness and carotid intima-media thickness: two independent markers of subclinical vascular damage in young adults? Eur J Clin Invest 33:949-954
6. Montecchi FR, Menzinger G, Lala A (2001) Carotid intimamedia thickness in patients with Type 2 diabetes and hypercholesterolemia. Diabetes Nutr Metab 14:58-61

7. Howard G, Sharrett AR, Heiss G et al (1993) Carotid artery intimal-medial thickness distribution in general population as evaluated by B-mode ultrasound. Stroke 24:1297-1304

8. Gariepy J, Salomon J, Denarie N et al (1998) Sex and topographic differences in associations between large-artery wall thickness and coronary risk profile in a French working cohort. The AXA Study. Arterioscler Thromb Vasc Biol 18:584-590

9. Bots ML, Dijk JM, Oren A, Grobbee DE (2002) Carotid intima-media thickness, arterial stiffness and risk of cardiovascular disease: current evidence. J Hypertens 20:2317-2325

10. Melidonis A, Kyriazis IA, Georgopali A et al (2003) Prognostic value of the carotid artery intima-media thickness for the presence and severity of coronary artery disease in type 2 diabetic patients. Diabetes Care 26:3189-3190

11. Ciccone M, Vettor R, Pannacciulli N et al (2001) Plasma leptin is independently associated with the intima-media thickness of the common carotid artery. Int J Obes Related Metab Disord 25:805-810

12. Wakabayashi I, Masuda H (2004) Relationship between obesity and atherosclerotic risk in elderly type 2 diabetic patients. Nippon Ronen Igakkai Zasshi 41:223-227 (Japanese with English abstract) 